\title{
Comportamento de Aedes albopictus e de Ae. scapularis adultos (D iptera: Culicidae) no Sudeste do Brasil
}

\section{Adults Aedes albopictus and Ae. scapularis behavior (Diptera: Culidae) in Southeastern Brazil}

O swaldo Paulo Forattinia, Iná Kakitani ${ }^{a}$, Roseli La Corte dos Santos ${ }^{b}$, Keilla M Kobayashi $^{b}$, Helene M U eno ${ }^{b}$ e Zoraida Fernandez ${ }^{b}$

${ }^{\text {aD }}$ epartamento de Epidemiologia da Faculdade de Saúde Pública da U niversidade de São Paulo. São Paulo, SP, Brasil. bNúcleo de Pesquisa Taxonômica e Sistemática em Entomologia M édica da Universidade de São Paulo (N U PTEM). São Paulo, SP, Brasil 


\title{
Comportamento de Aedes albopictus e de Ae. scapularis adultos (Diptera: Culicidae) no Sudeste do Brasil* Adults Aedes albopictus and Ae. scapularis behavior (Diptera: Culidae) in Southeastern Brazil
}

\author{
O swaldo Paulo Forattinia, Iná Kakitania, Roseli La Corte dos Santos ${ }^{b}$, Keilla M \\ Kobayashi' ${ }^{b}$ Helene M U eno ${ }^{b}$ e Zoraida Fernandez ${ }^{b}$
}

aD epartamento de Epidemiologia da Faculdade de Saúde Pública da U niversidade de São Paulo. São Paulo, SP, Brasil. ' $N$ úcleo de Pesquisa Taxonômica e Sistemática em Entomologia M édica da U niversidade de São Paulo (NUPTEM). São Paulo, SP, Brasil

\section{Descritores}

Aedes $^{\#}$. Ecologia de vetores ${ }^{\#}$. Insetos vetores. Culicidae. - Aedes albopictus. Aedes scapularis.

\section{Keywords}

Aedes $^{\#}$. Ecology, vector ${ }^{\#}$. Insect vectors. Culicidae. - Aedes albopictus. Aedes scapularis.

\section{Resumo}

\section{Objetivo}

Observar e comparar o comportamento das espécies de Aedes albopictus e de Ae. scapularis, na localidade de Pedrinhas, litoral sul do Estado de São Paulo, Brasil.

\section{Métodos}

As observações foram feitas de outubro de 1996 a janeiro de 2000. Foram realizadas coletas sistemáticas de formas adultas mediante a utilização de isca humana, aspirações ambientais e armadilha tipo Shannon. A domiciliação foi estimada pelo índice de Nuorteva e pela razão de sinantropia.

\section{Resultados}

Foram feitas 87 coletas diurnas, com a obtenção de 872 adultos fêmeas. As médias de Williams', multiplicadas por 100, foram de 118 e 21 para Ae. albopictus nos horários de $7 \mathrm{~h}$ às $18 \mathrm{~h}$ e de $18 \mathrm{~h}$ às $20 \mathrm{~h}$, respectivamente. Quanto a Ae. scapularis, foram de 100 e 106 nos mesmos períodos. Esse último revelou pico de atividade crepuscular vespertina. Na aspiração de abrigos, obteve-se o total de 1.124 espécimens, dos quais 226 Ae. albopictus e 898 Ae. scapularis. O período de janeiro a maio correspondeu ao de maior rendimento para ambos os mosquitos. Quanto à armadilha de Shannon, as coletas realizadas na mata revelaram a ausência de $A e$. albopictus. No que concerne à domiciliação, esse último mostrou os maiores valores de índices, enquanto Ae. scapularis revelou comportamento de tipo ubiquista.

Conclusões

Os resultados confirmam outras observações, permitindo levantar hipóteses. Em relação a Ae. scapularis, sugere-se que possa existir fenômeno de diapausa das fêmeas no período verão-outono, a qual cessaria no inverno-primavera quando então a atividade seria retomada. Quanto a Ae. albopictus, os dados sugerem que se trata de população em processo adaptativo ao novo ambiente.

\section{Abstract}

Objective

Aedes albopictus and Ae. scapularis were found living together in the Pedrinhas Village, Southeastern of São Paulo State, Brazil. This finding was a good opportunity
Correspondência para/Correspondence to:

Oswaldo Paulo Forattini

NUPTEM

Av. Dr. Arnaldo, 715

01246-904 São Paulo, SP, Brasil.

E-mail: opforati@usp.br
*Pesquisa subvencionada pela "Fundação de Amparo à Pesquisa do Estado de São Paulo (Fapesp)" (Processo Temático 95/0381-4)

Edição subvencionada pela Fapesp (Processo n. 00/01601-8).

Recebido em 7/4/2000. Aprovado em 26/7/2000. 


\section{to make observations about the mosquitoes' behavior.}

Methods

From October 1996 to January 2000 observations were carried out through systematic collections with human bait, environment aspirations and Shannon trap utilization. Synanthropy was estimated by the Nuorteva index and synanthropic ratios. Results

The 87 collections with human bait yield 872 females adults. Williams' means, multiplied by 100, were 118 and 21 for Ae. albopictus at the 7 AM-6PM and 6PM8PM hours, respectively, 100 and 106 for Ae. scapularis at the same timetable but there was an evening peak. Through environmental aspirations, a total of 1,124 adults samples was collected, 226 Ae. albopictus samples and 898 Ae. scapularis samples. The period between the months of January-May was the one with higher yield for both mosquitoes. There was no Ae. albopictus in the Shannon trap operated inside the adjacent forest. Regarding the sinanthropy, that culicid showed the higher index values, while Ae. scapularis was ubiquitous.

Conclusions

The data obtained allows to form the hypothesis that Ae. scapularis females may have a diapause phase in the resting places and after that period they will retake the hematophagy habit. That might explain the higher activity at the humen bait during the dry months, corresponding to the period of July-October.

\section{INTRO DU ÇÃO}

Para estudar o comportamento de mosquitos Culicidae em relação à população humana, há de se observá-los em áreas de transformação antrópica. Essa poderá ocorrer ao longo de gradação diversificada, desde a predominância da área natural até a profunda, na qual se dá a construção do meio artificial antrópico, tanto rural como urbano. No que concerne aos mecanismos de transmissão de infecções, tornase necessário identificar as populações culicídeas potencialmente vetoras as quais se mostram influenciadas por fatores diversos. Um deles é representado pela atração para exercer a hematofagia no hospedeiro humano. Nesse particular, são consideradas de grande utilidade as informações obtidas mediante o uso da isca humana. Outro fator consiste na freqüência do vetor aos meios peri e intradomiciliar. Nesse caso, a observação poderá ser feita mediante coletas, não apenas com a isca humana, mas também pelo processo de aspiração efetuado nesses ambientes.

Admite-se que condições diversas possam exercer influência nos resultados conseguidos com aquela isca humana. Algumas lhe são inerentes, enquanto outras dizem respeito aos mosquitos supostamente vetores. Há concordância quanto à associação entre as preferências hematófagas e a emissão de $\mathrm{CO}_{2}$ e de substâncias produzidas pela pele do hospedeiro. Para algumas espécies, a isca humana tenderia a atrair maior número de fêmeas oníparas do que grávidas (Ulloa et al, ${ }^{11} 1997$ ).

Na região do Vale do Ribeira, Sudeste do Estado de São Paulo, Brasil, a partir da segunda metade do decênio de 1990, tem-se assinalado de maneira signifi- cativa a presença de Aedes albopictus. Por sua vez, também se encontrou Ae. scapularis com densidade populacional apreciável e mesmo tendo sido observado com franca tendência à sinantropia (Forattini et al, ${ }^{4}$ 1995). Os hábitos hematófagos dessas espécies regionais, apesar de um tanto oportunistas, têm mostrado alguma tendência para mamíferos. Todavia, e, pelo menos ao que diz respeito à determinada população norte-americana de Ae. albopictus, o homem parece não ocupar lugar de destaque nessa preferência (Savage et al, ${ }^{9}$ 1993).

O encontro dessas populações na mesma área ensejou a possibilidade de observar a atividade hematófaga e a frequiência domiciliar de cada uma das espécies. E isso mediante comparações de comportamento, sediadas em núcleo destinado a sofrer alterações antrópicas de origem predominantemente turística.

\section{MÉTODOS}

\section{Área de estudo}

As observações foram levadas a efeito na vila das Pedrinhas, sede do Distrito de mesmo nome e pertencente ao município de Ilha Comprida. Situa-se no litoral sul do Estado de São Paulo, Brasil, e corresponde às coordenadas geográficas de $24^{\circ} 91^{\prime}$ de latitude sul e de $47^{\circ} 80^{\prime}$ de longitude oeste. Trata-se de pequeno núcleo com cerca de 400 habitantes e 231 edificações, de acordo com o levantamento efetuado em março de 2000. Dista, em linha reta, cerca de 25 $\mathrm{km}$ da sede municipal e 16 da cidade próxima de Cananéia. $\mathrm{O}$ acesso se faz pela praia, a partir da qual uma estrada de cerca de $4 \mathrm{~km}$ liga-a ao núcleo. Assim 
sendo, a vila de Pedrinhas localiza-se às margens do sistema lagunar interposto entre a ilha de Cananéia, na qual ela se situa, e o continente. $\mathrm{O}$ canal ali existente recebe o nome de Mar Pequeno (Figura 1).

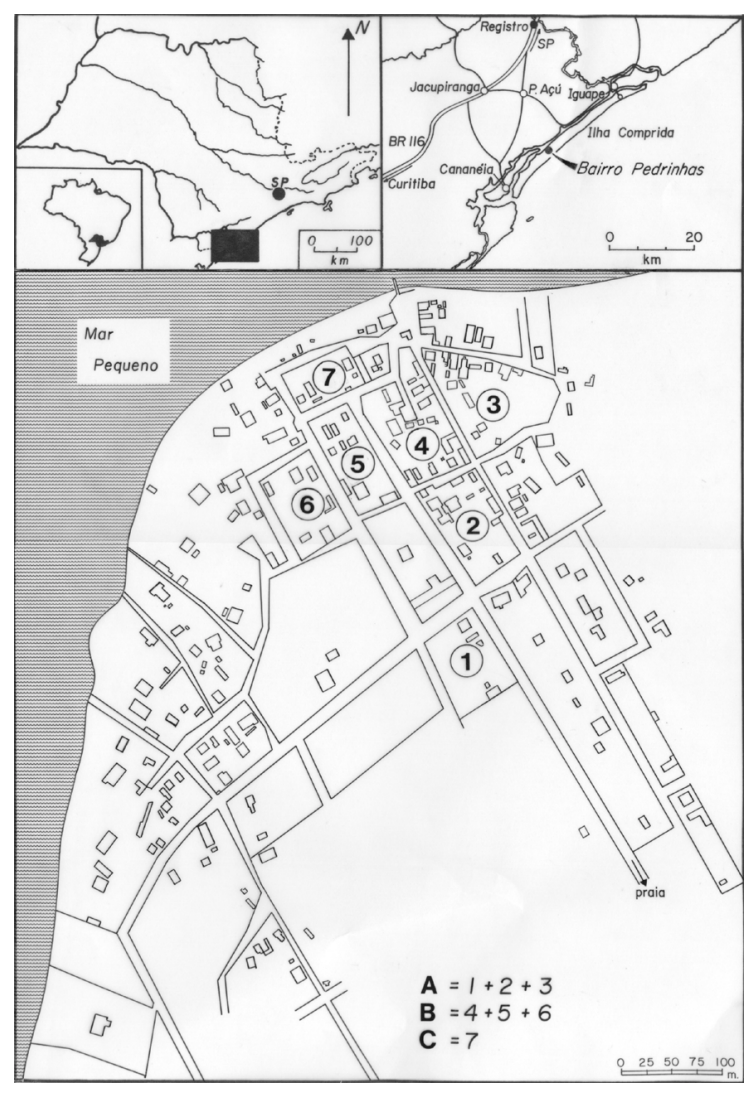

Figura 1 - Planta e localização geográfica da vila de Pedrinhas, Sudeste do Estado de São Paulo, Brasil. As áreas trabalhadas estão representadas por letras correspondentes às descrições constantes no texto.

O sistema supra-referido é dotado de dinâmica extremamente complexa, em boa parte influenciada pela atividade humana. Em vista disso, é comumente denominado de "Complexo Estuarino Lagunar de Iguape e Cananéia". Compreende as lagunas de águas salobras, as ilhas situadas em seu interior e os assentamentos humanos existentes na região, dos quais a vila de Pedrinhas constitui exemplo. Assim, pois, trata-se de ambiente tipicamente lagunar, no qual a Ilha Comprida desempenha o papel de barreira, separando-o do mar aberto, porém mantendo comunicação com esse pelas Barras. A sedimentação cenozóica recente é o principal fator de elaboração, em grande parte ocupada pelos depósitos da chamada Formação de Cananéia (Suguio, ${ }^{10}$ 1980; Petri \& Fulfaro ${ }^{8}$ 1983).

Ao longo do período das coletas, o macroclima apresentou as condições próprias do padrão regional, cujos dados foram fornecidos pelo Instituto Oceanográfico da Universidade de São Paulo, sediado em Cananéia.
As médias mensais das temperaturas e das precipitações atmosféricas registradas oscilaram de $15^{\circ} \mathrm{C} \mathrm{a} 38^{\circ} \mathrm{C}$ e de $36 \mathrm{~mm}$ a cerca de $600 \mathrm{~mm}$, respectivamente.

Na sua maior parte, a área de coleta esteve primitivamente coberta de vegetação natural representada pelas restingas. No caso particular da vila de Pedrinhas, trata-se da restinga interna e da mata (Dau, ${ }^{1}$ 1960), além do mangue que margeia o canal do Mar Pequeno. O núcleo foi instalado originariamente em terreno com disposição vegetal em mosaico, característica da mencionada restinga interna, além de solo com mata aberta contendo indivíduos xeromórficos, os quais podem atingir alturas de até 30 metros. Em ambas coberturas vegetais, pôde-se notar a abundante existência de bromélias, tanto terrestres quanto epífitas. Tais plantas são predominantemente de natureza silvestre, embora algumas tenham sido conservadas ao redor das casas, caracterizando assim a feição domiciliada (Forattini et al, ${ }^{5}$ 1998).

No que tange à ocupação humana, o caráter turístico, referido em parágrafo anterior, é o fenômeno da "segunda residência", amplamente disseminado nas áreas litorâneas paulistas. Em sua grande maioria, é representado por habitações de veraneio. No caso da vila de Pedrinhas, pouco mais de $35 \%$ das casas são permanentemente habitadas. Isso traz, de forma intermitente e correspondendo às temporadas, contingente populacional flutuante. Nos intervalos dessas épocas, as residências são ocupadas por pessoas desempenhando as funções de caseiros. A exemplo de outras regiões do litoral brasileiro, instala-se assim dinâmica capitalista de uso e da propriedade da terra, com a especulação imobiliária conseqüiente (Moraes, ${ }^{7}$ 1999).

\section{Isca humana (IH)}

Para a coleta pouso-homem ("human landing"), foi utilizado aspirador manual antes que a picada e a subseqüente sucção fossem levadas a efeito.

As coletas foram realizadas no peridomicílio de outubro de 1996 a janeiro de 2000. Foram selecionadas duas residências próximas uma à outra e uma delas habitada permanentemente. As capturas tiveram lugar em ritmo quinzenal, ou seja, em semanas alternadas, ocupando um dia de cada, durante o qual, e com o concurso de operador único, procedeu-se ao início das atividades às $7 \mathrm{~h}$. Os espécimes obtidos foram separados em lotes correspondentes às horas operadas e de acordo com os seguintes horários: de outubro de 1996 a janeiro de 1999, das 7h às 18h; e de fevereiro de 1999 a janeiro de 2000, das $7 \mathrm{~h}$ às $20 \mathrm{~h}$.

A adição de duas horas objetivou detectar o possí- 
vel início de atividades por parte de mosquitos com hábitos noturnos.

A partir da coleta efetuada em 7/10/1997, acrescentou-se, como rotina, a aspiração do ambiente no qual aquela foi realizada (IH-Asp). E isso após o término da captura e com a duração de $15 \mathrm{~min}$. A finalidade foi obter espécimes que deixaram de ser atraídos pela isca humana (IH).

\section{Aspiração (Asp)}

A captura de adultos foi feita nos locais por eles usados como abrigos. Para tanto, utilizou-se do aspirador de Nasci já empregado em outras observações levadas a efeito na mesma região (Forattini et $\mathrm{al}^{3}{ }^{3}$ 1987).

Para a execução dessas aspirações, selecionaramse dois conjuntos, cada um representado por três quarteirões, de maneira que o total deles foi numerado de 1 a 6 . Os conjuntos supracitados foram os seguintes, de acordo com os quarteirões englobados: $\mathrm{A}=1,2$ e $3 ; \mathrm{B}=4,5$ e 6 .

Em cada um desses agrupamentos realizaram-se as aspirações $A s p A$ e $A s p B$, em semana alternada mensal. Dessa forma, considerando-as globalmente, a aspiração ambiental foi executada em ritmo bimensal. A $A s p C$ foi realizada no quarteirão 7 no qual estavam incluídas as habitações que sediaram as coletas com a isca humana e a aspiração final (IH-Asp). Assim, além delas, as coletas consistiram nas aspirações peridomiciliar $(A s p C-P)$ e intradomiciliar (AspC-I). A Figura 1 mostra a localização dessas capturas.

No que concerne aos períodos e aos ritmos adotados, pode-se resumi-los da maneira seguinte:

\begin{tabular}{lcr}
\hline Coleta & Período & Ritmo \\
\hline AspA & $11 / 1996-1 / 2000$ & mensal \\
AspB & idem & mensal \\
AspC-P & $12 / 1996-1 / 2000$ & bimensal \\
AspC-I & $10 / 1997-1 / 1999$ & bimensal \\
\hline
\end{tabular}

\section{Armadilha de Shannon (Shan)}

Objetivando estabelecer comparações, foram feitas coletas com essa metodologia. A operação da ar- madilha obedeceu a ritmo quinzenal, em locais e períodos distintos, a saber:

\begin{tabular}{lcr}
\hline Coleta & Local (horário) & Período \\
\hline ShanA & mata $(15: 00-18: 00)$ & $10 / 1996-09 / 1997$ \\
ShanB & vila $(15: 00-18: 00)$ & $3 / 1998-01 / 1999$ \\
ShanC & vila $(17: 00-20: 00)$ & $2 / 1999-01 / 2000$ \\
\hline
\end{tabular}

\section{Domiciliação}

A análise teve como objetivo precípuo a estimativa do potencial sinantrópico das populações culicídeas focalizadas no presente trabalho. Para tanto, utilizouse o índice de Nuorteva $(s)$, bem como da razão de sinantropia $(s r)$, a qual foi estimada pela comparação dos resultados obtidos em várias coletas.

No que concerne aos dados conseguidos com a isca humana, usaram-se as médias de Williams' $\left(\overline{\mathrm{X}}_{\mathrm{w}}\right)$, cujos valores foram aproximados e multiplicados por 100, para obter cifras inteiras.

\section{RESULTADOS}

\section{Isca humana}

Ao longo de 87 coletas, 29 das quais prolongadas até às $20 \mathrm{~h}$, foram capturados 872 espécimens adultos fêmeas. A distribuição das médias de Williams' está representada na Tabela 1, de acordo com os horários dos períodos de coleta. Relacionando-se mensalmente a pluviosidade média registrada nos quatro anos, 1996-1999, obteve-se o gráfico representado pela Figura 2. As duas curvas indicam o período compreendente aos primeiros cinco meses anuais, janeiro a maio, como sendo o de maior produtividade de adultos de $A e$. albopictus. Por sua vez, o tempo que inclui os meses de julho a outubro parece ser o de maior produção de Ae. scapularis. Calculando-se as médias de Williams' correspondentes a esses dois períodos, e distribuídas pelas horas do dia nas quais se procedeu às coletas, obtiveram-se as curvas representadas no gráfico da Figura 3. Pode-se ver que ao longo do dia se mantém a maior freqüência por parte de Ae. albopictus. Contudo, no final da tarde, a de Ae. scapularis sobrepuja-a. Esse pico parece corresponder às horas nas quais ocorre o crepúsculo vespertino.

Tabela 1 - Distribuição das médias de Williams' $\left(\mathrm{X}_{\mathrm{w}}\right)$ de adultos fêmeas, coletados em isca humana em Pedrinhas, SP, de acordo com o horário da coleta.

\begin{tabular}{|c|c|c|c|c|c|c|}
\hline \multirow[b]{2}{*}{ Espécies } & \multirow{2}{*}{ Horas } & \multicolumn{2}{|c|}{ 7:00-18:00 } & \multicolumn{2}{|c|}{$18: 00-20: 00$} & \multirow{2}{*}{$\begin{array}{c}\text { Total } \\
\mathrm{n} \\
\end{array}$} \\
\hline & & $\mathrm{n}$ & $\left(X_{w} \cdot 100\right)$ & $\mathrm{n}$ & $\left(\bar{X}_{w} \cdot 100\right)$ & \\
\hline $\begin{array}{l}\text { Ae. albopictus } \\
\text { Ae. scapularis }\end{array}$ & & $\begin{array}{l}389 \\
381\end{array}$ & $\begin{array}{l}118 \\
100\end{array}$ & $\begin{array}{l}10 \\
92\end{array}$ & $\begin{array}{c}21 \\
106\end{array}$ & $\begin{array}{l}399 \\
473\end{array}$ \\
\hline Total & & 770 & - & 102 & - & 872 \\
\hline
\end{tabular}

$\mathrm{n}=$ número de espécimes 


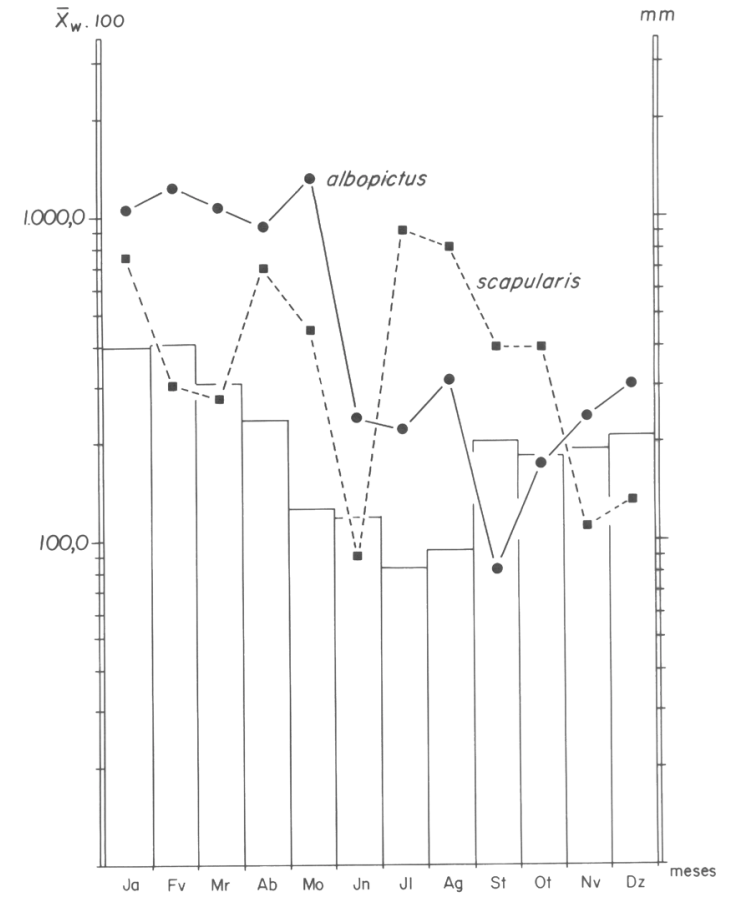

Figura 2 - Variação mensal das médias de Williams' $\left(\bar{X}_{\mathrm{W}} \bullet 100\right)$ obtidas com o emprego da isca humana, em Pedrinhas, SP. As barras representam as médias da pluviosidade (mm) no período 1996-1999.

Quanto às aspirações finais (IH-Asp), foram feitas em número de 61 ao longo do período, totalizando $15 \mathrm{~h} 25$. Nelas foram obtidos 101 adultos dos quais 22 (14 fêmeas e 8 machos) pertencentes a Ae. albopictus e 79 (60 fêmeas e 19 machos) a Ae. scapularis. Isso forneceu média horária total de 6,6, da qual 0,9 para as fêmeas do primeiro daqueles mosquitos e 3,9 para as do segundo.

\section{Aspirações (Asp)}

Considerando-se em conjunto as coletas mensais representadas por $A s p A$ e $A s p B$, os dados referentes às fêmeas foram de 99 para Ae. albopictus e de 441 para Ae. scapularis. Para os indivíduos masculinos observou-se proporção aproximada a essa (Tabela 2).

Com Ae. albopictus o maior rendimento nas coletas obedeceu ao padrão verificado para a isca humana, isto é, ao período de janeiro a maio. Todavia, esse fenômeno não se repetiu para Ae. scapularis,

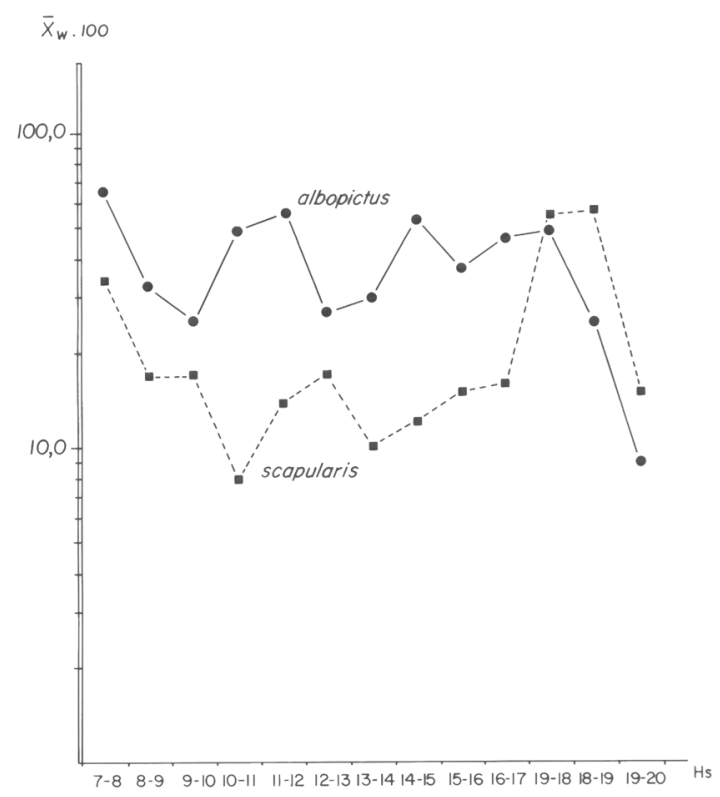

Figura 3 - Variação horária das médias de Williams' $\left(\bar{X}_{\mathrm{W}} \bullet 100\right)$ obtidas em coletas diurnas com o emprego da isca humana, em Pedrinhas, SP. O s dados dizem respeito aos períodos de maior produtividade de acordo com o gráfico da Figura 2, ou seja, de janeiro a maio para Ae. albopictus e de julho a outubro para Ae. scapularis.

ou seja, em relação aos meses de julho a outubro. Esse mosquito revelou-se mais freqüente nas coletas $A s p A$ e $A s p B$ nos mesmos meses de janeiro a maio. Praticamente a grande maioria dos espécimens foi coletada nesse período, e de acordo com os anos de observação:

\begin{tabular}{lrcrc}
\hline & 1997 & 1998 & \multicolumn{1}{c}{1999} & 2000 (jan) \\
\hline fêmeas & $38(38,0 \%)$ & $195(92,4 \%)$ & $115(93,5 \%)$ & 6 \\
machos & $9(28,1 \%)$ & $165(98,8 \%)$ & $73(78,5 \%)$ & 7 \\
\hline
\end{tabular}

Quanto às aspirações $A s p C$, como se referiu, foram feitas no peri $(A s p C-P)$ e no intradomicílio (AspC-I). Para tanto, foi utilizado conjunto de duas habitações no qual se levou a efeito a coleta com a isca humana. Os resultados totais obtidos estão apresentados na Tabela 2. Compulsando os dados mensais, não se pôde observar a ocorrência de variações acentuadamente diferentes. Exceto talvez a relativa ausência de ambos os mosquitos a partir de 6/1998 nas AspC-P. No que concerne aos resultados obti-

Tabela 2 - Número de espécimes coletados com as aspirações em Pedrinhas, SP.

\begin{tabular}{|c|c|c|c|c|c|c|c|c|c|c|c|c|c|}
\hline \multirow{3}{*}{$\begin{array}{l}\text { Espécies } \\
\text { Coletas }\end{array}$} & \multicolumn{4}{|c|}{ Ae. albopictus } & \multicolumn{4}{|c|}{ Ae. scapularis } & \multicolumn{4}{|c|}{ Total } & \multirow[b]{3}{*}{$\mathrm{n}$} \\
\hline & \multicolumn{2}{|c|}{ ९ ९ } & \multicolumn{2}{|c|}{$\sigma \sigma$} & \multicolumn{2}{|c|}{ ९ } & \multicolumn{2}{|c|}{$\sigma \sigma$} & \multicolumn{2}{|c|}{ १ 9} & \multicolumn{2}{|c|}{$\sigma \sigma$} & \\
\hline & $\bar{n}$ & $\%$ & $\mathrm{n}$ & $\%$ & $\mathrm{n}$ & $\%$ & $n$ & $\%$ & $\mathrm{n}$ & $\%$ & $\mathrm{n}$ & $\%$ & \\
\hline $\begin{array}{l}\text { AspA } \\
\text { AspB } \\
\text { AspC-P } \\
\text { AspC-I }\end{array}$ & $\begin{array}{r}49 \\
50 \\
39 \\
9\end{array}$ & $\begin{array}{r}33,4 \\
34,0 \\
26,5 \\
6,1\end{array}$ & $\begin{array}{r}25 \\
28 \\
23 \\
3\end{array}$ & $\begin{array}{r}31,6 \\
35,4 \\
29,1 \\
3,8\end{array}$ & $\begin{array}{r}224 \\
217 \\
99 \\
2\end{array}$ & $\begin{array}{r}41,3 \\
40,0 \\
18,2 \\
0,4\end{array}$ & $\begin{array}{r}175 \\
136 \\
45\end{array}$ & $\begin{array}{l}49,2 \\
38,2 \\
12,6\end{array}$ & $\begin{array}{r}273 \\
267 \\
138 \\
11\end{array}$ & $\begin{array}{r}39,6 \\
38,8 \\
20,0 \\
1,6\end{array}$ & $\begin{array}{r}200 \\
164 \\
68 \\
3\end{array}$ & $\begin{array}{r}45,9 \\
37,7 \\
15,6 \\
0,7\end{array}$ & $\begin{array}{r}473 \\
431 \\
206 \\
14\end{array}$ \\
\hline Total & 147 & 100,0 & 79 & 99,9 & 542 & 99,9 & 356 & 100,0 & 689 & 100,0 & 435 & 99,9 & 1.124 \\
\hline
\end{tabular}


Tabela 3 - Resultados obtidos com a utilização da armadilha de Shannon (Shan) em Pedrinhas.

\begin{tabular}{|c|c|c|c|c|c|c|c|c|c|c|c|c|c|c|c|}
\hline \multirow{3}{*}{$\begin{array}{l}\text { Espécies } \\
\text { Coletas }\end{array}$} & \multicolumn{7}{|c|}{ Ae. albopictus } & \multicolumn{5}{|c|}{ Ae. scapularis } & \multirow{2}{*}{\multicolumn{3}{|c|}{ Total }} \\
\hline & & 99 & & & $\sigma \quad \sigma$ & & & 9 9 & & & $\sigma \sigma$ & & & & \\
\hline & $n$ & $\%$ & $\mathrm{mh}$ & $\mathrm{n}$ & $\%$ & $\mathrm{mh}$ & $n$ & $\%$ & $\mathrm{mh}$ & $\mathrm{n}$ & $\%$ & $\mathrm{mh}$ & $n$ & $\%$ & $\mathrm{mh}$ \\
\hline $\begin{array}{l}\text { ShanA } \\
\text { ShanB } \\
\text { ShanC }\end{array}$ & $\begin{array}{l}62 \\
41\end{array}$ & $\begin{array}{l}6 \overline{-}, 2 \\
39,8\end{array}$ & $\begin{array}{l}\overline{0}, 5 \\
0,3\end{array}$ & $\begin{array}{l}- \\
4 \\
-\end{array}$ & - & $\begin{array}{l}- \\
\cdots \\
-\end{array}$ & $\begin{array}{l}238 \\
259 \\
644\end{array}$ & $\begin{array}{l}20,8 \\
22,8 \\
56,4\end{array}$ & $\begin{array}{l}1,7 \\
2,0 \\
4,5\end{array}$ & $\begin{array}{l}- \\
4 \\
1\end{array}$ & $\begin{array}{l}80,0 \\
20,0\end{array}$ & $\begin{array}{l}- \\
\ldots \\
\ldots\end{array}$ & $\begin{array}{l}238 \\
329 \\
686\end{array}$ & $\begin{array}{l}19,0 \\
26,3 \\
54,7\end{array}$ & $\begin{array}{l}1,7 \\
2,5 \\
4,8\end{array}$ \\
\hline Total & 103 & 100,0 & 0,3 & 4 & . & $\ldots$ & 1.141 & 100,0 & 2,7 & 5 & 100,0 & $\ldots$ & 1.253 & 100,0 & 3,0 \\
\hline
\end{tabular}

mh = média horária.

$\mathrm{n}=$ número de espécimes

dos com AspC-I, a presença dos dois culicídeos foi praticamente nula.

\section{Armadilha de Shannon (Shan)}

Foram coletados 1.253 espécimens, com a média horária correspondente a 3,0, cujos resultados estão expostos na Tabela 3. As fêmeas predominaram com mais de $99 \%$ dos exemplares capturados. Contudo, Ae. scapularis, com $91,7 \%$, ultrapassou as representantes de Ae. albopictus as quais perfizeram 8,3 desse total. Quanto ao horário das coletas, o das $17 \mathrm{~h}$ às $20 \mathrm{~h}$, e referente à ShanC, mostrou incremento dos espécimens coletados, o que correu por conta da freqüência de Ae. scapularis.

\section{Domiciliação}

Para o cálculo do índice de Nuorteva $(s)$, considerou-se como do ambiente domiciliar os dados obtidos com a isca humana, como referentes ao meio antrópico modificado os conseguidos com as armadilhas de Shannon (ShanB + ShanC) e os relativos ao ambiente primitivo, aqueles decorrentes do uso da ShanA. Assim sendo, em relação aos dois mosquitos, os resultados foram os seguintes:

\begin{tabular}{ccccc}
\hline & IH & ShanB+ShanC & ShanA & S \\
\hline Ae. albopicuts & $399(45,8 \%)$ & $103(10,1 \%)$ & - & $+50,8$ \\
Ae. scapularis & $473(54,2 \%)$ & $903(88,9 \%)$ & $238(100 \%)$ & $-1,35$ \\
\hline & 872 & 1.015 & 238 & \\
\hline
\end{tabular}

No que concerne à razão de sinantropia $(s r)$ entre as duas populações focalizadas, os resultados obtidos foram:

\section{Ae. albopictus}

$$
\mathrm{sr}=\frac{\mathrm{IH}}{\text { ShanB }+ \text { ShanC }}=4,5
$$

Ae. scapularis

$$
\mathrm{sr}=\frac{\mathrm{IH}}{\mathrm{ShanB}+\text { ShanC }}=0,6
$$

$$
\mathrm{sr}=\frac{\text { ShanB }+ \text { ShanC }}{\text { ShanA }}=0,9
$$

Comparando-se assim as populações desses dois mosquitos, pôde-se detectar a elevada sinantropia da de Ae. albopictus, a qual não foi encontrada no ambiente primitivo (ShanA). Por outro lado, a de Ae. scapularis mostrou-se freqüente tanto na mata adjacente como no meio domiciliar.

\section{DISCUSSÃO}

Face a tais resultados, chama a atenção o predomínio de Ae. scapularis em relação a Ae. albopictus. Procurando verificar possível associação com a pluviosidade, a primeira dessas espécies evidencia-se pelo fato de ser mais abundante em época de menor precipitação atmosférica. Assim sendo, a freqüência desse mosquito torna-se marcante no período de julho a outubro na isca humana. Por outro lado, ao longo do dia, revela-se a existência de pico correspondente às últimas horas da tarde, englobando o crepúsculo vespertino. A repetição desses dois fenômenos nas atuais pesquisas vieram confirmar o já observado na mesma região do Vale do Ribeira (Forattini et al, ${ }^{2}$ 1981).

Ainda em relação a Ae. scapularis, a explicação do maior comparecimento nos meses secos permanece ainda como questão a ser esclarecida. Comparando os dados com aqueles correspondentes a $A e$. albopictus, verifica-se que esses atingem valores mais expressivos nos meses chuvosos de janeiro a maio. Até certo ponto, pode-se entender isso pelo fato desse mosquito criar-se em recipientes, enquanto aquele o faz nas coleções do solo. A maior intensidade de precipitações atmosféricas faria com que se enchessem os recipientes, ao mesmo tempo em que as coleções líquidas no solo seriam "lavadas". Todavia, as coletas levadas a efeito mediante aspiração do ambiente vieram a trazer dados os quais possibilitam o levantamento de hipóteses outras. Assim, Ae. scapularis mostra-se mais abrigado no período de chuvas intensas. Pode-se argumentar que essas se tornam causa de maior mortalidade de adultos, além de limpar os locais no solo, passíveis de servi- 
rem de criadouros. Assim sendo, poder-se-ia supor que as fêmeas desse mosquito entrassem em diapausa no período. E isso até que as condições ambientais propiciassem a retomada das atividades. Tratar-seia, pois, de fenômeno cíclico em população univoltina cuja hematofagia seria exercida em época mais favorável do ano. Essa corresponderia aos meses de julho a outubro.

Quanto a Ae. albopictus, trata-se de espécie exótica a qual parece estar em fase de adaptação ao ambiente. Na região Sudeste do Brasil tudo leva a crer que a maior atividade corresponde ao período de janeiro a maio e no horário da manhã (Marques

\section{REFERÊN CIAS}

1. Dau L. Microclimas das restingas do sudeste do Brasil. IRestinga interna de Cabo Frio. Arq Museu $\mathrm{Nac}$ 1960;50:79-133.

2. Forattini OP, Gomes A de C, Santos JLF, Galati EAB, Rabello EX, Natal D. O bservações sobre atividade de mosquitos Culicidae em mata residual no Vale do Ribeira, S.Paulo, Brasil. Rev Saúde Pública 1981;15:557-86.

3. Forattini OP, Gomes A de C, N atal D, Kakitani I, $M$ arucci $D$. Freqüência domiciliar e endofilia de mosquitos Culicidae no Vale do Ribeira, São Paulo, Brasil. Rev Saúde Pública 1987;21:188-92.

4. Forattini OP, Kakitani I, Massad E, Marucci D. Studies on mosquitoes (Diptera: Culicidae) and enthropic environment. 9 - Synanthropy and epidemiological vector role of Aedes scapularis in South-Eastern Brazil. Rev Saúde Pública 1995;29:199-207.

5. Forattini OP, Marques GRAM, Kakitani I, Brito M de, Sallum MAM. Significado epidemiológico dos criadouros de Aedes albopictus em bromélias. Rev Saúde Pública 1998;32:186-8.
\& Gomes, ${ }^{6}$ 1997). As presentes observações confirmam esse comportamento.

No que concerne à domiciliação, torna-se evidente o elevado valor atingido por Ae. albopictus. Bem assim como a capacidade sinantrópica de Ae. scapularis, já assinalada em outras oportunidades (Forattini et al, ${ }^{4}$ 1995). A presença dessas duas populações em núcleo habitado pequeno e adjacente próximo ao ambiente natural primitivo enseja a possibilidade de transmissão de agentes infecciosos aos habitantes humanos e animais domésticos ali existentes. Esse aspecto tem particular interesse epidemiológico no caso das arboviroses.

6. M arques GRAM , Gomes A de C. Comportamento antropofílico de Aedes albopictus (Skuse) (Diptera: Culicidae) na região do Vale do Paraíba, Sudeste do Brasil. Rev Saúde Pública 1997;31:125-30.

7. Moraes ACR. Contribuições para a gestão da zona costeira do Brasil: elementos para uma geografia do litoral. São Paulo: Hucitec/EDU SP; 1999.

8. Petri S, Fúlvaro VJ. Geologia do Brasil. São Paulo: T.A.Q ueiroz/EDU SP; 1983.

9. Savage HM, Niebylski ML, Smith GC, Mitchell CJ, Craig Jr GB. Host-feeding patterns of Aedes albopictus (Diptera: Culicidae) at a temperate north american site. J Med Entomol 1993;30:27-34.

10. Suguio K. Rochas sedimentares. São Paulo: Edgard Blücher/EDU SP; 1980

11. Ulloa A, Rodríguez MH, Rodríguez AD, Roberts DR. A comparison of two collection methods for estimating abundance and parity of Anopheles albimanus in breeding sites and villages of Southern M exico. J Am Mosq Control Assoc 1997;13:238-44. 